\title{
Cosmic-ray fermion decay through tau-antitau emission with Lorentz violation
}

\author{
C. A. Escobar, J. P. Noordmans, and R. Potting \\ Departamento de Física, FCT, Universidade do Algarve, 8005-139 Faro, Portugal, \\ and CENTRA, Departamento de Física, Instituto Superior Técnico, Universidade de Lisboa, \\ Avenida Rovisco Pais 1, 1049-001 Lisboa, Portugal
}

(Received 28 April 2018; published 18 June 2018)

\begin{abstract}
We study $C P T$ and Lorentz violation in the tau-lepton sector of the Standard Model in the context of the Standard-Model Extension, parametrized by a coefficient which is thus far unbounded by experiment. We show that any nonzero value of this coefficient implies that, for sufficiently large energies, Standard Model fermions become unstable against decay due to the emission of a pair of tau-antitau leptons. We calculate the induced fermion energy-loss rate and we deduce the first limit on the Lorentz- and $C P T$-violation coefficient.
\end{abstract}

DOI: 10.1103/PhysRevD.97.115030

\section{INTRODUCTION}

Lorentz symmetry is a fundamental ingredient in both the Standard Model and general relativity. Nevertheless, various candidate theories of quantum gravity suggest Lorentz violation (LV) may occur at the Planck level [1]. Consequently, there have been extensive searches for Lorentz-violating signals. Many of these studies use a general effective-field-theory framework called the Standard-Model Extension (SME) [2,3]. It contains all LV gauge-invariant effective operators that can be built from the conventional Standard Model fields, coupled to vector and tensor coefficients that parametrize the LV. Within the SME, consistency requirements such as gauge invariance, stability and causality can be maintained [4]. The SME also contains all $C P T$-violating operators, because in any local interacting quantum field theory CPT violation (CPTV) implies LV [5]. The SME allows us to quantify, in the most general possible way, the exactness of Lorentz and CPT symmetry in the form of observational constraints on the Lorentz-violation coefficients (LVCs) [6]. Such restrictions on LV and CPTV may be used as a guide in finding the correct theory of quantum gravity.

It is generally safe to assume the LVCs, if nonzero, will be very small. Consequently, they will generally produce very small effects in certain physical quantities. However, there are also cases in which LVCs allow

Published by the American Physical Society under the terms of the Creative Commons Attribution 4.0 International license. Further distribution of this work must maintain attribution to the author(s) and the published article's title, journal citation, and DOI. Funded by SCOAP ${ }^{3}$. certain processes to take place that are normally forbidden. In earlier work [7] it was shown how a CPT and LV operator in the sector of weak gauge bosons results in the possibility that at sufficiently high energy fermions that couple to these gauge bosons can emit on-shell W bosons, resulting in their decay. As such emissions should be able to occur for normally stable particles such as the proton, this opens the possibility to bound the relevant SME coefficient by considering the fact that protons are present in ultrahigh-energy cosmic rays (UHECRs).

In this work we consider a similar decay process, in which an incoming (low-mass) fermion is taken to emit a tau-antitau lepton pair through a virtual gauge boson (a photon or a $Z$ boson), losing energy in the process. Normally this process is, of course, not allowed because of nonconservation of energy. For a hadron one might imagine it could occur if the emission provokes the decay of the hadron into other particles. Nevertheless, such a scenario is impossible for the proton, because baryon number conservation imposes that in the final decay products there has to be at least one (stable) baryon, which has at least the mass of the proton. In this paper we will assume that the propagation of the gauge boson is described by its usual Lorentz-symmetric Lagrangian, while the kinetic Lagrangian for the tau lepton is taken to include a $C P T$ and $\mathrm{LV}$ vector coefficient $b^{\mu}$ which is so far unbounded by experiment. As we show, the presence of this coefficient will change the dispersion relation of the tau and antitau leptons in such a way that the fermion decay becomes possible in phase space above a certain (large) threshold energy of the incoming fermion. This threshold energy is inversely proportional to the size of the components of the LV coefficient $b^{\mu}$, which we will suppose to be 
very small. In other words, we will assume the Earth-bound frame is concordant [4].

A bound on $b^{\mu}$ can be obtained by applying this process to the proton. The presence of protons in UHECRs implies their stability at least up until the energy for which they are observed. One crucial step is the demonstration that the decay rate associated with this process is sufficiently large to exclude the possibility that the proton would have survived the trip from their astrophysical source to the Earth even when its momentum exceeds the threshold for tau-antitau emission.

This paper is organized as follows. In Sec. II we analyze the kinetic Lagrangian of the tau lepton in the presence of the Lorentz- and $C P T$-violation coefficient $b^{\mu}$ and determine the dispersion relation and the properties of the spin states. In Sec. III we analyze the kinematics of the emission of a tau-antitau pair by an elementary fermion in the presence of a $b^{\mu}$ coefficient in the kinetic term of the tau lepton. In Sec. IV we evaluate the emission rate of this process in two regimes of the momentum for an incoming high-energy Dirac fermion. This result is extended to the case of a proton in Sec. V. Finally, observational data from UHECRs are used to produce a bound on the components of the $b^{\mu}$ coefficient.

\section{THE LORENTZ-VIOLATING MODEL}

We consider the following kinetic Lagrangian describing the Standard Model tau lepton:

$$
\mathcal{L}_{\mathrm{LV}}=\bar{\psi}\left(i \not \partial-\gamma^{5} \not \partial-m_{\tau}\right) \psi,
$$

where the constant four-vector $b^{\mu}$ parametrizes a LV and $C P T$-violating operator in the tau sector within the context of the Standard-Model Extension [2,3]. It can either be timelike, lightlike, or spacelike.

The energy-momentum tensor of the field $\psi$ is given by

$$
T^{\mu \nu}=\frac{\partial \mathcal{L}}{\partial\left(\partial_{\mu} \psi\right)} \partial^{\nu} \psi-\eta^{\mu \nu} \mathcal{L} .
$$

Since $\partial_{\mu} T^{\mu \nu}=0$ follows from Noether's theorem applied to invariance under spacetime translations, we have two conserved quantities: the Hamiltonian and the physical momentum:

$$
\begin{aligned}
& H=\int d^{3} x T^{00}=\int d^{3} x \bar{\psi}\left(i \vec{\gamma} \cdot \vec{\partial}+\gamma^{5} \not b+m_{\tau}\right) \psi, \\
& P^{i}=\int d^{3} x T^{0 i}=\int d^{3} x i \psi^{\dagger} \partial^{i} \psi=\int d^{3} x \pi \partial^{i} \psi,
\end{aligned}
$$

where

$$
\pi=\frac{\partial \mathcal{L}}{\partial\left(\partial_{0} \psi\right)}=i \psi^{\dagger}
$$

is the canonical momentum. From the Euler-Lagrange equation we get the following Lorentz-violating equation of motion for $\psi$ :

$$
\left(i \gamma^{\mu} \partial_{\mu}-b_{\mu} \gamma_{5} \gamma^{\mu}-m_{\tau}\right) \psi=0 .
$$

From this equation of motion, or from Eq. (3), we derive that

$$
i \partial^{0} \psi=\gamma^{0}\left(i \vec{\gamma} \cdot \vec{\partial}+m_{\tau}+\gamma^{5} \not b\right) \psi \equiv \hat{H}[i \vec{\partial}] \psi
$$

First, notice that $\hat{H}[i \vec{\partial}]$ is Hermitian. It therefore has real eigenvalues, i.e., all energies are real.

If we use the ansatz $\psi(x)=W(\vec{\lambda}) e^{-i \lambda \cdot x}$, the equation of motion for the spinors becomes $\left(\chi-\gamma^{5} \not b-m_{\tau}\right) W^{\alpha}(\vec{\lambda})=0$ with $\alpha \in\{1, \ldots, 4\}$, or equivalently

$$
\begin{aligned}
\gamma^{0}\left(\chi-\gamma^{5} \not b-m_{\tau}\right) W^{\alpha}(\vec{\lambda}) & =\left(\lambda^{0}-\hat{H}[\vec{\lambda}]\right) W^{\alpha}(\vec{\lambda}) \\
& \equiv \Lambda_{\alpha}(\lambda) W^{\alpha}(\vec{\lambda}),
\end{aligned}
$$

with $\Lambda_{\alpha}(\lambda)=\lambda^{0}-\omega_{\alpha}(\vec{\lambda})$, where $\omega_{\alpha}(\vec{\lambda})$ are the roots of the dispersion relation. This dispersion relation is given by

$$
\left(\lambda^{2}-m_{\tau}^{2}-b^{2}\right)^{2}-4(\lambda \cdot b)^{2}+4 \lambda^{2} b^{2}=\tilde{\Lambda}_{+}(\lambda) \tilde{\Lambda}_{-}(\lambda)=0 .
$$

Here

$$
\tilde{\Lambda}_{ \pm}(\lambda)=\lambda^{2}-m_{\tau}^{2}-b^{2} \pm 2 \sqrt{(\lambda \cdot b)^{2}-b^{2} \lambda^{2}},
$$

where $\tilde{\Lambda}_{+}(\lambda)$ and $\tilde{\Lambda}_{-}(\lambda)$ each have two nondegenerate roots. We will take the roots $\alpha=1,3$ to correspond to $\tilde{\Lambda}_{+}=0$, and the roots $\alpha=2,4$ to $\tilde{\Lambda}_{-}=0$. (Below, we will see that $\alpha=1,2$ will correspond to particle states, $\alpha=3,4$ to antiparticle states.) In fact, it is not hard to see that

$$
\prod_{\alpha=1}^{4} \Lambda_{\alpha}=\tilde{\Lambda}_{+} \tilde{\Lambda}_{-}
$$

The $W^{\alpha}(\vec{\lambda})$ satisfy the off-shell eigenvalue equation (8) (valid for arbitrary values of $\lambda^{\mu}$ ), as well as the on-shell equation:

$$
\hat{H}[\vec{\lambda}] W^{\alpha}(\vec{\lambda})=\omega_{\alpha}(\vec{\lambda}) W^{\alpha}(\vec{\lambda}) .
$$

Since the operator on the left-hand side of Eq. (8) is Hermitian, it has four orthogonal eigenvectors $W^{\alpha}(\vec{\lambda})$ :

$$
W^{\alpha}(\vec{\lambda})^{\dagger} W^{\beta}(\vec{\lambda})=\delta^{\alpha \beta} N_{\alpha}(\vec{\lambda}),
$$

with $N_{\alpha}(\vec{\lambda})$ a real and positive normalization factor to be chosen later. They satisfy the completeness relation 


$$
\sum_{\alpha=1}^{4} \frac{W^{\alpha}(\vec{\lambda}) W^{\alpha}(\vec{\lambda})^{\dagger}}{N_{\alpha}(\vec{\lambda})}=\mathbb{1} .
$$

Multiplying identity (14) by $\gamma^{0}$ from the left and the right and using Eq. (8) then yields

$$
\left(\chi-m_{\tau}-\gamma^{5} \not b\right)^{-1}=\sum_{\alpha=1}^{4} \frac{W^{\alpha}(\vec{\lambda}) \bar{W}^{\alpha}(\vec{\lambda})}{N_{\alpha}(\vec{\lambda}) \Lambda_{\alpha}(\lambda)},
$$

with $\bar{W}^{\alpha}(\vec{\lambda})=W^{\alpha}(\vec{\lambda})^{\dagger} \gamma^{0}$. Using the matrix identity $A^{-1}=$ $\operatorname{adj}(A) / \operatorname{det}(A)$, as well as the identity $\operatorname{det}\left(\chi-m_{\tau}-\gamma^{5} \not h\right)=$ $\prod_{\alpha=1}^{4} \Lambda_{\alpha}(\lambda)$, it then follows that

$$
\begin{aligned}
W^{\alpha}(\vec{\lambda}) \bar{W}^{\alpha}(\vec{\lambda}) & \left.=\frac{N_{\alpha}(\vec{\lambda}) \operatorname{adj}\left(\chi-m_{\tau}-\gamma^{5} \not b\right)}{\prod_{\beta \neq \alpha} \Lambda_{\beta}(\lambda)}\right]_{\lambda^{0}=\omega_{\alpha}(\vec{\lambda})} \\
& \left.=\frac{N_{\alpha}(\vec{\lambda}) \operatorname{adj}\left(\chi-m_{\tau}-\gamma^{5} \not b\right)}{4\left(\lambda^{2}-m_{\tau}^{2}+b^{2}\right) \lambda^{0}-8(b \cdot \lambda) b^{0}}\right\rfloor_{\lambda^{0}=\omega_{\alpha}(\vec{\lambda})},
\end{aligned}
$$

where the last identity follows from taking the derivative of both sides of Eq. (11) with respect to $\lambda_{0}$. One can also verify that

$$
\begin{aligned}
\operatorname{adj}\left(\chi-m_{\tau}-\gamma^{5} \not \partial\right)= & \left(\chi \chi m_{\tau}-\gamma^{5} \not \not\right) \\
& \times\left(\lambda^{2}-m_{\tau}^{2}-b^{2}-2 i \gamma^{5} \sigma^{\mu \nu} b_{\mu} \lambda_{\nu}\right)
\end{aligned}
$$

with $\sigma^{\mu \nu}=\frac{i}{2}\left[\gamma^{\mu}, \gamma^{\nu}\right]$. Notice that it follows easily from the equation of motion that

$$
2 i \gamma^{5} \sigma^{\mu \nu} b_{\mu} \lambda_{\nu} W^{\alpha}(\vec{\lambda})=-\left(\lambda^{2}-m_{\tau}^{2}-b^{2}\right) W^{\alpha}(\vec{\lambda}) .
$$

Therefore, the spinors $W^{\alpha}(\vec{\lambda})$ are eigenstates, with eigenvalues \pm 1 , of the $C P T$-violating operator

$$
\mathcal{P}=\frac{i \gamma^{5} \sigma^{\mu \nu} b_{\mu} \lambda_{\nu}}{\sqrt{(\lambda \cdot b)^{2}-\lambda^{2} b^{2}}},
$$

where all quantities are put on the relevant mass shell. For purely timelike $b^{\mu}, \mathcal{P}$ can be seen to correspond to $\operatorname{sgn}\left(b^{0}\right)$ times the helicity operator. We take the spinors to satisfy

$$
\mathcal{P} W_{\alpha}(\vec{\lambda})=-(-1)^{\alpha} W_{\alpha}(\vec{\lambda}), \quad(\alpha=1, \ldots, 4) .
$$

We now take the normalizations for the spinors to be

$$
N^{\alpha}(\vec{\lambda})= \begin{cases}\left|\frac{\partial \tilde{\Lambda}_{+}(\lambda)}{\partial \lambda^{0}}\right|_{\lambda^{0}=\omega_{\alpha}(\vec{\lambda})} & \text { for } \alpha=1,3 ; \\ \left|\frac{\partial \tilde{\Lambda}_{-}(\lambda)}{\partial \lambda^{0}}\right|_{\lambda^{0}=\omega_{\alpha}(\vec{\lambda})} \mid & \text { for } \alpha=2,4 .\end{cases}
$$

Since the denominator in Eq. (16) is equal to $\frac{\partial}{\partial \lambda^{0}}\left(\tilde{\Lambda}_{+}(\lambda) \tilde{\Lambda}_{-}(\lambda)\right)$, we see that

$$
\begin{aligned}
& W^{\alpha}(\vec{\lambda}) \bar{W}^{\alpha}(\vec{\lambda}) \\
& = \begin{cases}\left.\frac{\operatorname{adj}\left(\chi-m_{\tau}-\gamma^{5} \not \not\right)}{2\left(\lambda^{2}-m_{\tau}^{2}-b^{2}\right)}\right|_{\lambda^{0}=\omega_{\alpha}(\vec{\lambda})} & \text { for } \alpha=1,2 ; \\
-\left.\frac{\operatorname{adj}\left(\chi-m_{\tau}-\gamma^{5} \not b\right)}{2\left(\lambda^{2}-m_{\tau}^{2}-b^{2}\right)}\right|_{\lambda^{0}=\omega_{\alpha}(\vec{\lambda})} & \text { for } \alpha=3,4 .\end{cases}
\end{aligned}
$$

By using Eq. (17) and the equation of motion it is then easy to see that

$$
\bar{W}^{\alpha}(\vec{\lambda}) W^{\alpha}(\vec{\lambda})= \begin{cases}2 m_{\tau} & \text { for } \alpha=1,2 \\ -2 m_{\tau} & \text { for } \alpha=3,4\end{cases}
$$

The dispersion relation (9) is invariant under $\lambda \rightarrow-\lambda$. So for each positive solution $\lambda^{0}=\omega(\vec{\lambda})$ there is another negative solution $\lambda^{0}=-\omega(-\vec{\lambda})$. As usual we take these to correspond to particle and antiparticle energies. We label the particle energies by a $u$ and the antiparticle energies by a $v$, while we differentiate between helicity states by a \pm sign, corresponding to the eigenvalue of the operator $\mathcal{P}$. It follows that

$$
\begin{aligned}
& E_{+}^{u}(\vec{p})=E_{-}^{v}(\vec{p})=\omega_{1}(\vec{p}, b)=-\omega_{3}(-\vec{p}, b), \\
& E_{-}^{u}(\vec{p})=E_{+}^{v}(\vec{p})=\omega_{2}(\vec{p}, b)=-\omega_{4}(-\vec{p}, b) .
\end{aligned}
$$

The corresponding redefined spinors are $u_{+}(\vec{p})=W^{1}(\vec{p})$, $u_{-}(\vec{p})=W^{2}(\vec{p}), v_{+}(\vec{p})=W^{4}(-\vec{p})$, and $v_{-}(\vec{p})=W^{3}(-\vec{p})$.

Notice that the labels of the antiparticle states in (24) have flipped sign because of the sign change under the identification $\lambda^{\mu} \rightarrow-p^{\mu}$, in accordance with the fact that $\mathcal{P}$ flips sign when $\lambda^{\mu}$ does. This obviates the fact that corresponding particle and antiparticle states have different energies, in accordance with the $C P T$-violating nature of the Lagrangian $\mathcal{L}_{\mathrm{LV}}$.

To first nontrivial order in the LVC, the particle energies (24) can be easily evaluated and, for arbitrary $b^{\mu}$, they are given by

$$
\begin{aligned}
& E_{ \pm}^{u}= E_{\mp}^{v}=\sqrt{\vec{\lambda}^{2}+m_{\tau}^{2}} \\
& \mp \frac{\sqrt{\left(b_{0} \sqrt{\vec{\lambda}^{2}+m_{\tau}^{2}}-\vec{\lambda} \cdot \vec{b}\right)^{2}-b^{2} m_{\tau}^{2}}}{\sqrt{\vec{\lambda}^{2}+m_{\tau}^{2}}} .
\end{aligned}
$$

Exact expressions for the energy can be written down for the cases $b_{0}=0, \vec{b}=0$ or $b^{0}= \pm|\vec{b}|$ (see [2], where also explicit expressions for the corresponding eigenspinors are derived), but we will not need them in this work. 


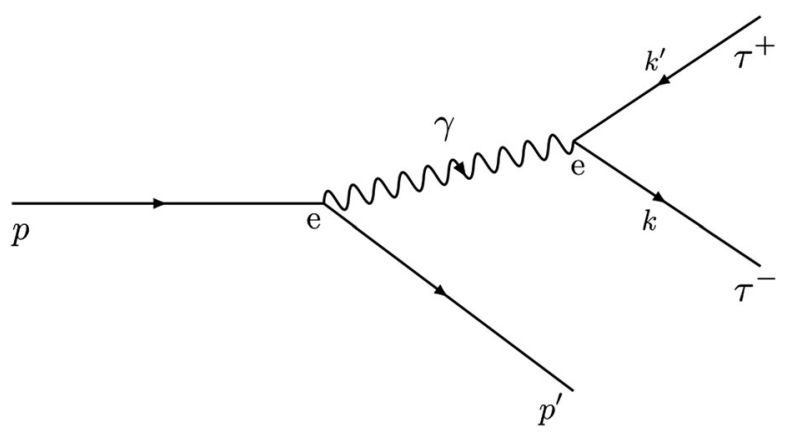

FIG. 1. Feynman diagram for the photon-mediated emission process of a $\tau^{-}, \tau^{+}$pair with Lorentz-violating dispersion relation by a Dirac fermion of incoming momentum $p$ and outgoing momentum $p^{\prime}$.

\section{KINEMATICS}

We aim to calculate the rate at which a high-energy Dirac fermion with mass $m$ emits a pair tau-antitau described by the Lagrangian (1). As a first step, we take the process to be mediated by a photon. The corresponding Feynman diagram is indicated in Fig. 1. In the standard Lorentzsymmetric case, this process is forbidden by energymomentum conservation. However, we will assume that the tau and antitau leptons in the final state are described by the $C P T$ and LV Lagrangian (1). This implies the modified dispersion relations defined by Eq. (24). In the following we will see that these imply the possibility of the emission process to proceed if the momentum of the incoming fermion exceeds a certain threshold value, at least if we take the final spin states such that the tau and antitau have spacelike four-momentum. This threshold value will turn out to be inversely proportional to the absolute value of the LVC $b^{\mu}$. Assuming $b^{\mu}$ to be very small in the Earth-bound frame (which we will assume in this work), it then follows that the emission process can only take place for very large incoming momenta.

Let us denote the momenta of the incoming and outgoing fermion by $p$ and $p^{\prime}$, and those of the tau and antitau by $k$ and $k^{\prime}$. We parametrize the three-momenta as follows:

$$
\begin{array}{ll}
\vec{p}=\left(\begin{array}{c}
0 \\
0 \\
p
\end{array}\right), & \vec{p}^{\prime}=\left(\begin{array}{c}
-k_{x}-k_{x}^{\prime} \\
-k_{y}-k_{y}^{\prime} \\
\lambda p
\end{array}\right), \\
\vec{k}=\left(\begin{array}{c}
k_{x} \\
k_{y} \\
\alpha p
\end{array}\right), & \vec{k}^{\prime}=\left(\begin{array}{c}
k_{x}^{\prime} \\
k_{y}^{\prime} \\
\beta p
\end{array}\right),
\end{array}
$$

with

$$
0<\alpha, \beta, \lambda<1 \quad \text { and } \quad \alpha+\beta+\lambda=1,
$$

assuring three-momentum conservation. The condition of energy conservation $p_{0}^{\prime}+k_{0}+k_{0}^{\prime}=p_{0}$ will impose a threshold value on the incoming momentum $p$. In order to see how this comes about, we write

$$
\begin{gathered}
p_{0}=\sqrt{p^{2}+m^{2}} \approx p+\frac{m^{2}}{2 p}, \\
p_{0}^{\prime}=\sqrt{\lambda^{2} p^{2}+\left(k_{x}+k_{x}^{\prime}\right)^{2}+\left(k_{y}+k_{y}^{\prime}\right)^{2}+m^{2}} \\
\geq \sqrt{\lambda^{2} p^{2}+m^{2}} \approx \lambda p+\frac{m^{2}}{2 \lambda p} .
\end{gathered}
$$

Similarly, by using the LV dispersion relation (10) we can estimate

$$
\begin{aligned}
& k_{0 \pm} \geq \alpha p+\frac{m_{\tau}^{2}}{2 \alpha p}-(-1)^{\alpha_{k}} \xi_{b, k}, \\
& k_{0 \pm}^{\prime} \geq \beta p+\frac{m_{\tau}^{2}}{2 \beta p}-(-1)^{\alpha_{k^{\prime}}} \xi_{b, k^{\prime}} .
\end{aligned}
$$

Here the parameter $\xi_{b, k}$ is of the order of the size of the components of the LV coefficient $b^{\mu}$. More precisely,

$$
\xi_{b, k}=\left|b_{0}-\right| \vec{b}\left|\cos \left(\theta_{k b}\right)\right|,
$$

where $\theta_{k b}$ is the angle between the vectors $\vec{k}$ and $\vec{b}$. A similar identity holds for $\xi_{b, k^{\prime}}$. We see from (32) that $\xi_{b, k}$ depends on $b^{\mu}$ and the relative orientation of $\vec{k}$ and $\vec{b}$. The parameters $\alpha_{k}$ and $\alpha_{k^{\prime}}$ take the values 1 or 2, indicating the helicity state of the tau and antitau (see Sec. II).

Using the estimates (28)-(31) condition $p_{0}^{\prime}+k_{0}+k_{0}^{\prime}=$ $p_{0}$ yields the relation

$$
(-1)^{\alpha_{k}} \xi_{b, k}+(-1)^{\alpha_{k^{\prime}}} \xi_{b, k^{\prime}} \geq \frac{m^{2}}{2 p}\left[\frac{1}{\lambda}-1+\frac{m_{\tau}^{2}}{m^{2}}\left(\frac{1}{\alpha}+\frac{1}{\beta}\right)\right] .
$$

As the right-hand side of (33) is positive, this condition can only be satisfied if the same is true for the left-hand side. For this reason, we will choose the spin states of the tau and antitau such that $(-1)^{\alpha_{k}} \xi_{b, k}=\left|\xi_{b, k}\right|$ and $(-1)^{\alpha_{k^{\prime}}} \xi_{b, k^{\prime}}=$ $\left|\xi_{b, k^{\prime}}\right|$. It is also possible to take the smaller of the two terms with the opposite sign (assuming that the sum remains positive). However, this channel only kicks in at a higher threshold momentum, and therefore we will not consider it in detail [8].

Noting that the expression in square brackets in (33) is bounded from below due to conditions (27) it follows that $p$ has to exceed a certain threshold value $p_{\text {th }}$. In order to determine $p_{\text {th }}$, note that the equalities in Eqs. (29)-(31) can only be satisfied if the transverse ( $x$ and $y$ ) components of the outgoing momenta are all taken to vanish. Moreover, for $p$ to attain its minimum value, the values of $\alpha$ and $\beta$ need to be chosen such that the expression in square 
brackets in (33) attains its minimum value. Taken together these considerations imply that $p=p_{\text {th }}$ if

$$
k_{x}=k_{x}^{\prime}=k_{y}=k_{y}^{\prime}=0, \quad \alpha=\beta=\frac{m_{\tau}}{m+2 m_{\tau}} .
$$

Thus, at threshold the outgoing tau and antitau leptons move in exactly the same direction as the incoming fermion, and thus all momenta are collinear. This in turn means

$$
\theta_{k b}=\theta_{k^{\prime} b}=\theta_{p b}=\theta_{p^{\prime} b} \quad(\text { at threshold }),
$$

and thus

$$
\xi_{b, k}=\xi_{b, k^{\prime}} \equiv \xi_{b, p} \quad \text { (at threshold) }
$$

Combining Eqs. (33), (34) and (36) it then follows that

$$
p_{\text {th }}=\frac{m_{\tau}\left(m_{\tau}+m\right)}{\left|\xi_{b, p}\right|} .
$$

Above threshold, conditions (34) are no longer satisfied. In that case, the transverse momenta can become nonzero, and the parameters $\alpha$ and $\beta$ fluctuate around their threshold values. We can then write, instead of Eqs. (29)-(31),

$$
\begin{gathered}
p_{0}^{\prime} \approx \lambda p+\frac{\left(k_{x}+k_{x}^{\prime}\right)^{2}+\left(k_{y}+k_{y}^{\prime}\right)^{2}+m^{2}}{2 \lambda p} \\
k_{0 \pm} \approx \alpha p+\frac{k_{x}^{2}+k_{y}^{2}+m_{\tau}^{2}}{2 \alpha p}-(-1)^{\alpha_{k} \xi_{b, k},} \\
k_{0 \pm}^{\prime} \approx \beta p+\frac{k_{x}^{\prime 2}+k_{y}^{\prime 2}+m_{\tau}^{2}}{2 \beta p}-(-1)^{\alpha_{k^{\prime}}} \xi_{b, k^{\prime}},
\end{gathered}
$$

which are valid as long as the longitudinal components of the momenta are much larger than the transverse ones. This is certainly the case for momenta just above threshold. Relation (33) then becomes

$$
\begin{aligned}
(-1)^{\alpha_{k}} \xi_{b, k}+(-1)^{\alpha_{k^{\prime}}} \xi_{b, k^{\prime}} & \\
\approx & \frac{\left(k_{x}+k_{x}^{\prime}\right)^{2}+\left(k_{y}+k_{y}^{\prime}\right)^{2}+m^{2}}{2 \lambda p}-\frac{m^{2}}{2 p} \\
& +\frac{k_{x}^{2}+k_{y}^{2}+m_{\tau}^{2}}{2 \alpha p}+\frac{\left(k_{x}^{\prime}\right)^{2}+\left(k_{y}^{\prime}\right)^{2}+m_{\tau}^{2}}{2 \beta p} .
\end{aligned}
$$

It will be useful for the following to determine the energy loss of the fermion with momentum $p>p_{\text {th }}$. In order to do so, we consider the "brick-wall" observer frame in which the four-momentum transfer $q=k+k^{\prime}=p-p^{\prime}$ imparted on the incoming fermion is purely spacelike, and thus no energy, only three-momentum is transferred. That such a frame exists can be seen from the fact that both the tau and antitau must have spacelike momentum for the emission to be possible [7]. In this frame the tau dispersion relation dips to negative energies for a certain momentum range.

By analyzing the process in this frame and transforming back to the concordant frame, one finds that the incoming fermion suffers an energy loss of at least

$$
\Delta E_{\min }=\frac{m_{\tau}^{2}}{\left|\xi_{b, p}\right|} \sim p_{\text {th }}
$$

Another interesting result that can be extracted is that the three-momentum transfer in the brick-wall frame is of order

$$
\frac{m_{\tau}^{2}}{\gamma\left|\xi_{b, p}\right|}
$$

where $\gamma$ equals the gamma factor associated with the Lorentz transformation to the brick-wall frame. At threshold the denominator in (43) is of order $m_{\tau}$, of the same order or larger than the fermion rest mass. On the other hand, for ultrahigh momenta $p \gg p_{\text {th }}$ the product $\gamma\left|\xi_{b, p}\right|$ will generally be much larger than $m_{\tau}$, yielding a very small (nonrelativistic) momentum transfer. In this case the brick-wall frame corresponds practically to the fermion rest frame.

\section{EMISSION RATE}

In order to be able to draw relevant physical conclusions about the emission process, we need to estimate the rate of emission. The decay rate is given by

$$
\begin{aligned}
\Gamma= & \frac{1}{2 p_{0}} \int \frac{d^{3} k}{(2 \pi)^{3} \Lambda^{\prime}(k)} \frac{d^{3} k^{\prime}}{(2 \pi)^{3} \Lambda^{\prime}\left(k^{\prime}\right)} \frac{d^{3} p^{\prime}}{(2 \pi)^{3} 2 p_{0}^{\prime}} \\
& \times|\mathcal{M}|^{2}(2 \pi)^{4} \delta^{(4)}\left(p-p^{\prime}-k-k^{\prime}\right),
\end{aligned}
$$

where the squared matrix element $|\mathcal{M}|^{2}$ is summed (averaged) over the final (initial) fermion, but not over the final tau and antitau states. The unconventional factors

$$
\Lambda^{\prime}(p)=\frac{\partial \Lambda_{ \pm}(p)}{\partial p^{0}}
$$

in the denominator define a (positive-definite) normalization in which the phase space and the matrix element are separately observer Lorentz invariant [9], i.e., invariant under simultaneous Lorentz transformations of the momenta and the LV four-vector. Explicit observer Lorentz covariance of the formalism will allow us to transform to convenient observer frames later on.

The matrix element corresponding to the tree level process mediated by the photon is given by

$i \mathcal{M}=\bar{u}\left(p^{\prime}\right)\left(i e \gamma^{\mu}\right) u(p) \frac{-i \eta_{\mu \nu}}{q^{2}} \bar{u}_{\tau}\left(k^{\prime}\right)\left(-i e \gamma^{\nu}\right) v_{\tau}(k)$. 
Here $u(p)$ and $u\left(p^{\prime}\right)$ are conventional Dirac spinors, $q=k+k^{\prime}=p-p^{\prime}$ is the transferred momentum, while $u_{\tau}\left(k^{\prime}\right)$ and $v_{\tau}(k)$ are described by the Lorentz and $C P T$ violating kinetic Lagrangian (1). It follows from (46) that the emission rate (44) can be written as

$$
\Gamma=\frac{1}{2 p_{0}} \int \frac{d^{3} k}{(2 \pi)^{3} \Lambda^{\prime}(k)} \frac{d^{3} k^{\prime}}{(2 \pi)^{3} \Lambda^{\prime}\left(k^{\prime}\right)} \frac{8 \pi e^{4}}{q^{4}} L_{\tau}^{\mu \nu} W_{\mu \nu},
$$

where

$$
L_{\tau}^{\mu \nu}=\operatorname{Tr}\left[\bar{u}_{\tau}\left(k^{\prime}\right) \gamma^{\mu} v_{\tau}(k) \bar{v}_{\tau}(k) \gamma^{\nu} u_{\tau}\left(k^{\prime}\right)\right]
$$

and

$W_{\mu \nu}=\frac{1}{8 \pi} \int \frac{d^{3} p^{\prime}}{(2 \pi)^{3} 2 p_{0}^{\prime}} L_{\mu \nu}^{\text {fermion }}(2 \pi)^{4} \delta^{(4)}\left(p-p^{\prime}-k-k^{\prime}\right)$

with

$$
L_{\mu \nu}^{\text {fermion }}=\frac{1}{2} \sum_{\text {spins }} \operatorname{Tr}\left[\bar{u}\left(p^{\prime}\right) \gamma_{\mu} u(p) \bar{u}(p) \gamma_{\nu} u\left(p^{\prime}\right)\right]
$$

As it turns out, evaluating the decay rate for general values of the incoming momentum is technically prohibitively complicated. Fortunately, it is sufficient for our purposes to estimate the order of magnitude of the rate. We have been able to evaluate the rate in two regimes: (a) when the incoming fermion momentum $p$ is just above threshold, and (b) in the asymptotic regime, when the incoming fermion momentum is much larger than the threshold value.

We will first consider case (a), corresponding to the conditions (34) on the momenta of the particles. Just above threshold, the matrix element squared $|\mathcal{M}|^{2}$ associated with the Feynman diagram in Fig. 1 does not vary strongly. Thus, we can evaluate its value at threshold and obtain

$$
\begin{aligned}
\Gamma \approx & \left.\frac{e^{4}}{(2 \pi)^{5}} \frac{1}{2 p_{0}}\left[\frac{1}{\Lambda^{\prime}(k)} \frac{1}{\Lambda^{\prime}\left(k^{\prime}\right)} \frac{1}{2 p_{0}^{\prime}}|\mathcal{M}|^{2}\right]\right|_{\mathrm{th}} \\
& \times \int d^{3} k d^{3} k^{\prime} d^{3} p^{\prime} \delta^{(4)}\left(p-p^{\prime}-k-k^{\prime}\right),
\end{aligned}
$$

where "th" is defined by the conditions in Eq. (34). By using the fact that the tau and antitau satisfy the modified dispersion relations (39) and (40), as well as the following identity for the spinor bilinears,

$u_{\tau}^{i}(k) \bar{u}_{\tau}^{i}(k)=\frac{\left(\not k+m-\gamma^{5} \not b\right)\left(k^{2}-m^{2}-b^{2}-2 i \gamma^{5} \sigma^{\mu \nu} b_{\mu} k_{\nu}\right)}{2\left(k^{2}-m^{2}-b^{2}\right)}$, and

$v_{\tau}^{i}(k) \bar{v}_{\tau}^{i}(k)=\frac{\left(\not k-m+\gamma^{5} \not h\right)\left(k^{2}-m^{2}-b^{2}+2 i \gamma^{5} \sigma^{\mu \nu} b_{\mu} k_{\nu}\right)}{2\left(k^{2}-m^{2}-b^{2}\right)}$,

which follow from Eqs. (22) and (17), one can derive that

$$
\begin{aligned}
& {\left.\left[\frac{1}{\Lambda^{\prime}(k)} \frac{1}{\Lambda^{\prime}\left(k^{\prime}\right)} \frac{1}{2 p_{0}^{\prime}}|\mathcal{M}|^{2}\right]\right|_{\text {th }}} \\
& \quad=\left.\frac{2}{p^{3}}\left(\frac{m_{\tau}}{m}\right)^{2} \frac{1}{(\alpha \beta)^{2}}\right|_{\text {th }} \\
& \quad=\frac{2}{p^{3}} \frac{\left(m+2 m_{\tau}\right)^{4}}{m^{2} m_{\tau}^{2}}+\mathcal{O}\left(\frac{\xi^{2}}{p^{3} m^{2}}, \frac{m}{p^{4}}\right) .
\end{aligned}
$$

Moreover, one can show that

$$
\begin{gathered}
\int d^{3} k d^{3} k^{\prime} d^{3} p^{\prime} \delta^{(4)}\left(p-p^{\prime}-k-k^{\prime}\right) \\
=p^{3} \frac{m^{11 / 2} m_{\tau}^{3}}{\left(m+2 m_{\tau}\right)^{13 / 2}} \pi^{3} R^{4}
\end{gathered}
$$

where

$$
R^{2}=\mp \frac{4\left|\xi_{b, p}\right| p}{m^{2}}-\frac{4 m_{\tau}\left(m+m_{\tau}\right)}{m^{2}}
$$

so that

$$
\Gamma=\frac{2 \pi^{3} e^{4}}{(2 \pi)^{5}} \frac{m^{\frac{7}{2}} m_{\tau}}{\left(m+m_{\tau}\right)^{\frac{5}{2}}} \frac{R^{4}}{p} .
$$

Taking $p=a p_{\text {th }}$, it then follows that

$$
\Gamma \approx \frac{e^{4}\left|\xi_{b, p}\right|}{\pi^{2}} \frac{m_{\tau}^{2}}{\sqrt{m\left(m+m_{\tau}\right)^{3}}}(a-1)^{2} \theta(a-1) .
$$

Now let us consider case (b) for which the scale of the incoming fermion momentum $p$ is much larger than the threshold momentum for tau-antitau emission, or, equivalently, that $\left|\xi_{b, p}\right| \gg m_{\tau}\left(m_{\tau}+m\right) / p$. In this case, we see from the energy conservation relation (41) that we can expect that the transverse momenta of the outgoing particles should scale as $\sqrt{\left|\xi_{b, p}\right| p}$. For this reason we introduce the rescaled, dimensionless transverse momentum variables

$$
\tilde{k}_{x, y}=\frac{k_{x, y}}{\sqrt{\left|\xi_{b, p}\right| p}}, \quad \tilde{k}_{x, y}^{\prime}=\frac{k_{x, y}^{\prime}}{\sqrt{\left|\xi_{b, p}\right| p}} .
$$

Note that the transverse momentum components of the outgoing fermion can be expressed in terms of $\tilde{k}_{x, y}$ and $\tilde{k}_{x, y}^{\prime}$ by three-momentum conservation. 
In order to perform the calculation of the emission rate below, we now make the approximation that the identities (35) hold approximately in the asymptotic $\left(p \gg p_{\text {th }}\right)$ limit. This will be the case because the transverse $(x, y)$ components of $\vec{k}$ and $\vec{k}^{\prime}$ scale as the square root of the incoming momentum $p$, while the parallel $(z)$ component is proportional to $p$. Thus, the tau and antitau are emitted in a small forward cone along the incoming momentum. This means that we can take $\xi_{b, k} \approx \xi_{b, k^{\prime}} \approx \xi_{b, p}$ as fixed quantities in the dispersion relation.

Taking now the high- $p$ limit, maintaining terms at lowest order in $1 / p$, it follows that

$$
\begin{aligned}
|\mathcal{M}|^{2}= & \frac{16 m_{\tau}^{2} e^{4}(1-\alpha-\beta)}{\alpha \beta} \\
& \times \frac{\left|\xi_{b, p}\right| p K\left(1+(1-\alpha-\beta)^{2}\right)+m^{2}(\alpha+\beta)^{4}}{\left(\left|\xi_{b, p}\right| p K+m^{2}(\alpha+\beta)^{2}\right)^{2}}
\end{aligned}
$$

where we have introduced

$$
K=\left(\tilde{k}_{x}+\tilde{k}_{x}^{\prime}\right)^{2}+\left(\tilde{k}_{y}+\tilde{k}_{y}^{\prime}\right)^{2} .
$$

The expression for the asymptotic decay rate becomes

$$
\Gamma=\frac{e^{4} m_{\tau}^{2}\left|\xi_{b, p}\right|^{2} p^{3}}{(2 \pi)^{5}} \int \frac{d \alpha d \beta d \tilde{k}_{x} d \tilde{k}_{y} d \tilde{k}_{x}^{\prime} d \tilde{k}_{y}^{\prime}}{\alpha^{2} \beta^{2}} \frac{\left|\xi_{b, p}\right| p K\left(1+(1-\alpha-\beta)^{2}\right)+m^{2}(\alpha+\beta)^{4}}{\left(\left|\xi_{b, p}\right| p K+m^{2}(\alpha+\beta)^{2}\right)^{2}} \delta\left(p-p^{0}-k^{0}-k^{\prime 0}\right) .
$$

The integral over the transverse momenta can now be performed, and the resulting expression can be cast into the form

$$
\begin{aligned}
\Gamma= & \frac{2 e^{4} m_{\tau}^{2}}{(2 \pi)^{3} p} \int_{a_{1}}^{a_{2}^{-1}-1} d z\left(\frac{z}{1+z}\right)\left[-\frac{\ln \left(a_{2}(1+z)\right)}{2}+\ln \left(\sqrt{1-\frac{a_{1}}{z}}+\sqrt{1-\frac{a_{1}}{z}-a_{2}(1+z)}\right)\right] \\
& \times\left[-1+\frac{1}{2}\left(\frac{(z+1)^{2}+z^{2}}{z(1+z)}\right) \ln \left(\frac{z}{a_{1}}\right)\right]+\mathcal{O}\left(\frac{1}{p}\right)
\end{aligned}
$$

where we introduced the parameters

$$
a_{1}=\frac{m^{2}}{4\left|\xi_{b, p}\right| p}, \quad a_{2}=\frac{m_{\tau}^{2}}{\left|\xi_{b, p}\right| p}
$$

which tend to zero in the large- $p$ limit. For the case in which $m$ and $m_{\tau}$ are of the same order, both $a_{1}$ and $a_{2}$ are of order $p_{\text {th }} / p$.

While exact analytic evaluation of the $z$-integral in (63) is not feasible, one can deduce that the dominant contribution is of the form

$$
\frac{-\ln \left(a_{2}\right)}{a_{2}}\left(C_{1}+C_{2} \ln \left(a_{1} a_{2}\right)\right)
$$

for some dimensionless constants $C_{1}$ and $C_{2}$ [here we excluded the prefactors in (63)]. Numerical fitting of the $z$-integral in (63) to the formula (65) confirms that an excellent fit can be obtained. In Table I we list fitted values for $C_{1}$ and $C_{2}$ as a function of the ratio $a_{1} / a_{2}=m^{2} /\left(4 m_{\tau}^{2}\right)$. In obtaining the fit, we took $a=p / p_{\text {th }}$ distributed logarithmically along the range $10^{2}<a<10^{10}$. It follows that

$$
\Gamma \sim \frac{2 e^{4}\left|\xi_{b, p}\right|}{(2 \pi)^{3}}\left(-\ln \left(a_{2}\right)\right)\left(C_{1}+C_{2} \ln \left(a_{1} a_{2}\right)\right) .
$$

We see that the asymptotic form of the rate is proportional to $\left|\xi_{b, p}\right|$ times a quadratic expression in terms of $\ln (a)$.
In conclusion, a charged fermion will start emitting tauantitau pairs if its momentum $p$ exceeds a certain threshold value $p_{\text {th }}$. The emission rate is of the form

$\Gamma=\frac{e^{4}\left|\xi_{b, p}\right|}{2 \pi^{2}} G(a) \theta(a-1)=8 \alpha^{2}\left|\xi_{b, p}\right| G(a) \theta(a-1)$,

(with $\alpha$ the fine-structure constant) for a function $G(a)$ that satisfies

$G(a) \approx \begin{cases}\frac{2 m_{\tau}^{2}}{\sqrt{m\left(m+m_{\tau}\right)^{3}}(a-1)^{2},} & \text { if } a-1 \ll 1 ; \\ \frac{1}{2 \pi}\left(-\ln \left(a_{2}\right)\right)\left(C_{1}+C_{2} \ln \left(a_{1} a_{2}\right)\right), & \text { if } a \gg 1 .\end{cases}$

TABLE I. Fitted values of the parameters $C_{1}$ and $C_{2}$ in formula (65) as a function of the ratio $a_{1} / a_{2}$. The value $a_{1} / a_{2}=$ 0.138875 corresponds to taking for $m$ the proton mass. The value $a_{1} / a_{2}=2.5$ corresponds to taking for $m 10$ times the tau mass.

\begin{tabular}{lcr}
\hline \hline$a_{1} / a_{2}$ & $C_{1}$ & \multicolumn{1}{c}{$C_{2}$} \\
\hline 0.025 & 2.5211 & 0.00759 \\
0.138875 & 1.9721 & -0.00060 \\
0.25 & 1.7969 & -0.00341 \\
1.00 & 1.4097 & -0.01004 \\
2.5 & 1.1738 & -0.01442 \\
\hline \hline
\end{tabular}


From the expressions (58) and (66) we find that if $\mathcal{O}\left(\left|\xi_{b, p}\right|\right) \sim 10^{-10} \mathrm{GeV}$, corresponding to the bound we will find later on, the typical decay time (as measured in the Earth-bound frame) is

$$
t_{p} \sim 10^{-11} \mathrm{~s} .
$$

Moreover, it was shown at the end of the previous section that the emission process implies an energy loss of at least $m_{\tau}^{2} /\left(2\left|\xi_{b, p}\right|\right) \sim p_{\text {th }}$. It follows that for such values of $\left|\xi_{b, p}\right|$ all fermions in a decay cascade will fall below threshold within at most $a \times 10^{-11} \mathrm{~s}$.

\section{THE PROTON}

The analysis in the previous sections applies to an incoming fundamental charged Dirac fermion. In fact, of more practical interest to us will be the case of an incoming composite particle such as the proton. In applying the formalism developed in the previous sections to an incoming proton, a few adaptations are necessary.

First of all, the tensor $W_{\mu \nu}$ in expression (49) now applies to the photon coupling to the proton and becomes

$$
W^{\mu \nu}=\frac{1}{8 \pi} \sum_{\sigma} \sum_{X}\left\langle\mathrm{p}(p, \sigma)\left|J^{\nu}(-q)\right| X\right\rangle\left\langle X\left|J^{\mu}(q)\right| \mathrm{p}(p, \sigma)\right\rangle,
$$

where $|\mathrm{p}(p, \sigma)\rangle$ is a proton state with momentum $p$ and spin $\sigma, J^{\mu}(p)$ is the hadronic current, and $\mathbb{Z}_{X}$ represents a sum over the possible hadronic final states $X$ along with the corresponding integrations over phase space.

Insight into the final hadronic state can be gained from the discussion at the end of Sec. III. As it was argued there, for incoming momenta far above the threshold $p_{\text {th }}$ (that is, for $a \gg 1$ ), the momentum transfer $\vec{k}+\vec{k}^{\prime}$ in the rest frame of the fermion will be tiny. This means the impact of the tau-antitau emission on the proton will be small so that it will remain intact. That is, the final state $|X\rangle$ corresponds to a (ground-state) proton. The applicable hadron current $J^{\mu}$ then can be written with the usual proton structure functions:

$J^{\mu}(q)=e \bar{u}\left(p^{\prime}\right)\left[F_{1}\left(q^{2}\right) \gamma^{\mu}+\frac{\kappa}{2 m} F_{2}\left(q^{2}\right) i \sigma^{\mu \nu} q_{\nu}\right] u(p)$,

where the proton anomalous magnetic moment $\kappa=1.79$. Also, as $q$ will be tiny, we can take the limits $F_{1}(0)=$ $F_{2}(0)=1$. Thus, the only change with respect to the calculation of the rate as compared to the case of a fundamental Dirac fermion will be the $F_{2}$ term. However, as the latter is proportional to the momentum transfer components $q_{\nu}$, it can be expected to be very small compared to the first term, and can be safely ignored. We conclude that for ultrahigh proton momenta $p \gg p_{\text {th }}$, the emission rate is given by expression (44) and that the energy of the proton will cascade down as described at the end of Sec. IV.

As we saw in Sec. III, for momenta close to the threshold for emission, the momentum transfer in the brick-wall frame will become of the order of the proton rest mass. As a consequence, it is more adequate to consider the tau-antitau emission as a (deeply) inelastic process, resulting in the breakup of the proton. In this case we can use the parton model to evaluate $W^{\mu \nu}$ in expression (70). This essentially involves calculating the decay rate of an elementary quark that carries a fraction $x$ of the longitudinal proton momentum. We can thus use many of the results obtained for the elementary fermion rate. For a pedagogical introduction to parton-model calculations, we refer to Ref. [10].

The resulting emission rate in the context of the parton model becomes

$\Gamma=\frac{e^{4}\left|\xi_{b, p}\right|}{2 \pi^{2}} \sum_{q} \int_{0}^{1} d x\left(f_{q}(x)+\bar{f}_{q}(x)\right) \tilde{G}_{q}^{e e}(a x) \theta(a x-1)$.

Here the functions $f_{q}(x)$ and $\bar{f}_{q}(x)$ are the parton distributions functions (PDFs) for the quarks and antiquarks of flavor $q$, respectively, representing the probability of finding a quark with momentum fraction $x$ inside the proton. The PDFs are assumed to be independent of $p^{2}$, which is a good approximation to leading order in the strong coupling constant. The function $G_{q}(a x)$ corresponds to the absolute value squared of the first term in (73), and is equal to the function (68) with the substitution $m \rightarrow x m$, and an extra multiplication by the square of the quark charge fraction.

As the integral over $x$ in Eq. (72) runs up to 1, the maximum momentum of the parton involved in the emission of the tau-antitau pair equals that of the incoming proton itself. As a consequence the threshold proton momentum for tau-antitau emission will be given by formula (37), where $m$ is to be identified with the proton mass. At such values of $x$ close to 1, the proton PDFs for valence quarks decay to zero approximately as a constant times $(1-x)^{c_{q}}$ with $c_{u} \approx 4$ and $c_{d} \approx 5$ [11]. As a consequence, the integral over $x$ in Eq. (72) yields decay rates just above threshold that are suppressed as compared to the situation in which the proton would have been an elementary particle.

Finally, we note that in the above we only considered the emission process in which the tau-antitau emission is mediated by a virtual photon. In fact, there is the additional possibility that the emission takes place with an intermediate $Z$ boson instead (see Fig. 2). This means that the transition amplitude $\mathcal{M}$ is given by the sum of two terms: 


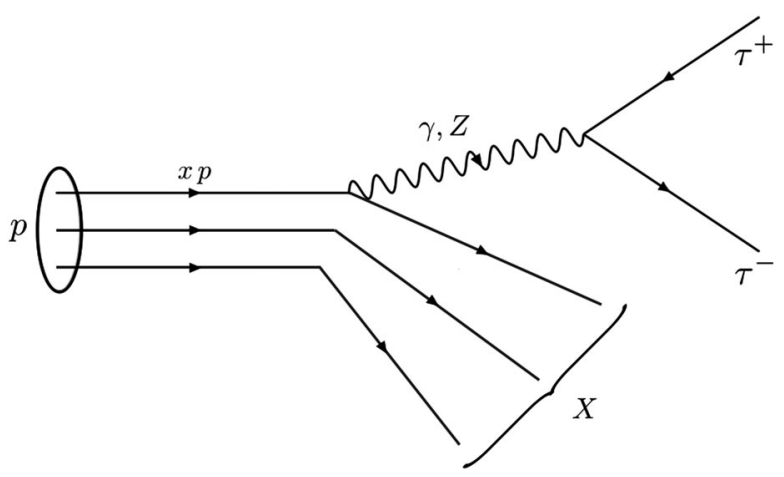

FIG. 2. Feynman diagram for the emission process of a tauantitau pair by a proton of incoming momentum $p$, mediated by a photon or a $Z$ boson.

$$
\begin{aligned}
i \mathcal{M}= & \left\langle X\left|i e J_{\mathrm{EM}}^{\mu}(q)\right| p(p, \sigma)\right\rangle \frac{-i \eta_{\mu \nu}}{q^{2}} \bar{u}_{\tau}\left(k^{\prime}\right)\left(-i e \gamma^{\nu}\right) v_{\tau}(k) \\
& +\left\langle X\left|i g J_{Z}^{\mu}(q)\right| p(q, \sigma)\right\rangle \frac{-i \eta_{\mu \nu}}{q^{2}-m_{Z}^{2}} \\
& \times \bar{u}_{\tau}\left(k^{\prime}\right)\left(-i \frac{g}{2} \gamma^{\nu}\right)\left(c_{V}^{\tau}-c_{A}^{\tau} \gamma^{5}\right) v_{\tau}(k) .
\end{aligned}
$$

Here $J_{\mathrm{EM}}^{\mu}$ corresponds to the electromagnetic current considered in (70) and in the previous sections, while $J_{Z}^{\mu}$ applies to the current coupling to the $Z$ boson. In the context of the parton model, the emission rate will now be of the form

$$
\begin{aligned}
\Gamma= & \frac{\left|\xi_{b, p}\right|}{2 \pi^{2}} \sum_{q} \int_{0}^{1} d x\left(f_{q}(x)+\bar{f}_{q}(x)\right) \\
& \times\left(e^{4} \tilde{G}_{q}^{e e}(a x)+g^{4} \tilde{G}_{q}^{g g}(a x)+e^{2} g^{2} \tilde{G}_{q}^{e g}(a x)\right) \theta(a x-1),
\end{aligned}
$$

where the function $\tilde{G}_{q}^{e e}(a x)$ is the same as $G_{q}(a x)$ in Eq. (72), while the terms with $\tilde{G}_{q}^{g g}$ and $\tilde{G}_{q}^{e g}$ correspond to the absolute value squared of the second term in (73), and a cross (interference) term, respectively. They can be expected to be of the same general form as $G_{q}^{e e}(a x)$. For our purposes it is not necessary to compute them explicitly, as we will only need a lower bound on the emission rate. It is sufficient to note that the additional terms are independent expressions proportional to the independent quantities $g^{4}$ and $e^{2} g^{2}$, and thus a cancellation would imply an extreme fine-tuning between $G_{q}^{e e}, G_{q}^{g g}$ and $G_{q}^{e g}$ (the latter would have to be negative).

In fact, note that in the asymptotic limit $p \gg p_{\text {th }}$ the momentum transfer $q^{\mu}$ in the brick-wall frame is very small. This means that the $Z$-boson propagator will be very suppressed compared to the photon propagator, which implies in turn that the decay rate will be dominated by the photon exchange diagram for $p \gg p_{\text {th }}$.

\section{OBSERVATIONAL LIMITS FROM ULTRAHIGH-ENERGY COSMIC RAYS}

We can obtain a bound on $\left|\xi_{b, p}\right|$ by observing that a cosmic-ray proton that has an energy below threshold has zero probability to disintegrate by tau-antitau emission, and can thus reach Earth unimpeded. However, with an energy above threshold, the proton can emit tau-antitau pairs, possibly disintegrating eventually, until all protons in the decay cascade have fallen below threshold. This means that it cannot reach Earth if its mean free path is much smaller than the distance $D$ from its source to Earth.

Since many ultrahigh-energy cosmic-ray (UHECR) particles with energies above $57 \mathrm{EeV} \equiv|\vec{p}|_{\text {obs }}$ have been observed, coming more or less from all directions [12], we can take it as a first estimate for the lower bound for $E_{\mathrm{th}}$. It follows that

$$
\left|\xi_{b, p}\right|<\frac{m_{\tau}\left(m_{\tau}+m\right)}{|\vec{p}|_{\text {obs }}} \approx 8.5 \times 10^{-11} \mathrm{GeV} .
$$

This bound can only be relaxed if the mean free path of protons above threshold is not much smaller than $D$. From Eq. (72) we see that the mean lifetime of protons (in Earth's frame) $t_{p}$ is still proportional to $\left|\xi_{b, p}\right|^{-1}$, but is enhanced, mainly by the minute values of the PDFs at large $x$. A very conservative estimation that comes from comparing to the elementary-fermion decay time (69) (involving only photon exchange) gives a mean free path of

$$
L \simeq c t_{p} \times 10^{15} \approx 3 \times 10^{9} \mathrm{~km}
$$

which is of the order of the size of the solar system. Clearly, in such a scenario, protons with an energy above threshold will not be able to reach Earth from any viable UHECR source. We thus obtain a bound on all four components of the LVC:

$$
\left|b^{\mu}\right|<8.5 \times 10^{-11} \mathrm{GeV} .
$$

We note that we have to assume that at least some of the detected UHECRs are protons and that these have a sufficient spread in arrival direction. Although the mass content of the UHECRs, in particular at high energies, is still uncertain [13], it seems very unlikely that such a significant low-mass component is completely absent. Also, considering the above-mentioned observations regarding the spread in arrival directions of the UHECRs, it is reasonable to assume that such a spread also applies to its proton component.

\section{CONCLUSION}

In this paper, we investigated Lorentz and $C P T$ violation in the electroweak sector. In particular, we focused on a hitherto unbounded LVC in the tau sector of the Standard-Model Extension. Considering the emission of 
tau-antitau pairs by high-energy particles in the context of UHECR observations leads to a new bound on the coupling $b^{\mu}$ in the tau sector of about $10^{-10} \mathrm{GeV}$. Our method relies on the fact that any hypothetical Lorentz violation in the tau sector parametrized by the $b^{\mu}$ coefficient turns a process that is normally forbidden in phase space possible for sufficiently high momentum. It is worth pointing out that formula (75) can be applied to the muon and the electron sectors as well, under the substitutions $m_{\tau} \rightarrow m_{\mu}$ and $m_{\tau} \rightarrow m_{e}$. This leads to the noncompetitive bounds $1.9 \times$ $10^{-12}$ and $8.4 \times 10^{-15} \mathrm{GeV}$ for the components of the $b^{\mu}$ parameter in the muon sector and the electron sector, respectively. However, we do expect that it should be possible to derive new and/or competitive bounds on other SME coefficients, either in the tau sector or for other particles, by applying this method.

\section{ACKNOWLEDGMENTS}

This work is supported in part by the Fundação para a Ciência e a Tecnologia of Portugal (FCT) through Projects No. UID/FIS/00099/2013 and No. SFRH/BPD/101403/ 2014 and Program No. POPH/FSE. C. A. E. was supported by a CONACyT Postdoctoral Grant No. 234745.
[1] V. A. Kostelecký and S. Samuel, Spontaneous breaking of Lorentz symmetry in string theory, Phys. Rev. D 39, 683 (1989); V. A. Kostelecky and R. Potting, CPT and strings, Nucl. Phys. B359, 545 (1991); J. R. Ellis, N.E. Mavromatos, and D. V. Nanopoulos, Search for quantum gravity, Gen. Relativ. Gravit. 31, 1257 (1999); R. Gambini and J. Pullin, Nonstandard optics from quantum space-time, Phys. Rev. D 59, 124021 (1999); C. P. Burgess, J. M. Cline, E. Filotas, J. Matias, and G. D. Moore, Loop generated bounds on changes to the graviton dispersion relation, J. High Energy Phys. 03 (2002) 043.

[2] D. Colladay and V. A. Kostelecký, $C P T$ violation and the standard model, Phys. Rev. D 55, 6760 (1997).

[3] D. Colladay and V. A. Kostelecký, Lorentz-violating extension of the standard model, Phys. Rev. D 58, 116002 (1998).

[4] V. A. Kostelecký and R. Lehnert, Stability, causality, and Lorentz and CPT violation, Phys. Rev. D 63, 065008 (2001).

[5] O. W. Greenberg, CPT Violation Implies Violation of Lorentz Invariance, Phys. Rev. Lett. 89, 231602 (2002).

[6] V. A. Kostelecky and N. Russell, Data tables for Lorentz and CPT violation, Rev. Mod. Phys. 83, 11 (2011); V. A. Kostelecky and N. Russell, Data Tables for Lorentz and CPT Violation, 2018 edition, arXiv:0801.0287v11

[7] D. Colladay, J. P. Noordmans, and R. Potting, Cosmic-ray fermion decay by emission of on-shell $\mathrm{W}$ bosons with $C P T$ violation, Phys. Rev. D 96, 035034 (2017).

[8] It suffices to note that for sufficiently large momentum the inclusion of this extra channel would lead to an increased value for the emission rate.
[9] D. Colladay, P. McDonald, J. P. Noordmans, and R. Potting, Covariant quantization of $C P T$-violating photons, Phys. Rev. D 95, 025025 (2017).

[10] M. E. Peskin and D. V. Schroeder, An Introduction to Quantum Field Theory (Addison-Wesley, Reading, MA, 1995).

[11] D. Stump, J. Huston, J. Pumplin, W. K. Tung, H. L. Lai, S. Kuhlmann, and J. F. Owens, Inclusive jet production, parton distributions, and the search for new physics, J. High Energy Phys. 10 (2003) 046; S. Alekhin, K. Melnikov, and F. Petriello, Fixed target Drell-Yan data and NNLO QCD fits of parton distribution functions, Phys. Rev. D 74, 054033 (2006); J. F. Owens, J. Huston, C. E. Keppel, S. Kuhlmann, J. G. Morfin, F. Olness, J. Pumplin, and D. Stump, Impact of new neutrino deep inelastic scattering and Drell-Yan data on large-x parton distributions, Phys. Rev. D 75, 054030 (2007).

[12] A. Aab et al. (Pierre Auger Collaboration), Searches for anisotropies in the arrival directions of the highest energy cosmic rays detected by the Pierre Auger Observatory, Astrophys. J. 804, 15 (2015); T. Abu-Zayyad et al. (Telescope Array Collaboration), Correlations of the arrival directions of ultrahigh energy cosmic rays with extragalactic objects as observed by the Telescope Array experiment, Astrophys. J. 777, 88 (2013).

[13] A. Aab et al. (Pierre Auger Collaboration), Depth of maximum of air-shower profiles at the Pierre Auger Observatory. II. Composition implications, Phys. Rev. D 90, 122006 (2014); R. U. Abbasi et al., Study of Ultra-High Energy Cosmic Ray composition using Telescope Array's Middle Drum detector and surface array in hybrid mode, Astropart. Phys. 64, 49 (2015). 